\title{
442 - Neurocognitive markers of passive suicidal ideation in late life depression
}

Joshua T. Jordan, Ph.D., Christina F. Chick, Ph.D., Camarin E. Rolle, B.S., B.A., Nathan Hantke, Ph.D., Christine E. Gould, Ph.D., Julie Lutz, Ph.D., Makoto Kawai, M.D., Isabelle Cotto, B.S., Rosy Karna, M.S., Sophia Pirog, B.A., Michelle Berk, Ph.D., Keith Sudheimer, Ph.D., Ruth O’Hara, Ph.D., \& Sherry A. Beaudreau, Ph.D.

Aims: Late life suicide is an international public health crisis, yet the mechanisms underlying late life suicide risk are far less understood compared to younger age groups. Executive dysfunction is widely documented in late life depression (LLD), and cognitive flexibility and inhibition are specifically hypothesized as vulnerabilities for suicide risk. There is some evidence that LLD patients with suicidal ideation or attempt suicide have worse executive dysfunction than LLD patients that do not; however, it is unknown whether these differences exist in Passive Suicidal Ideation (PSI), which may be an important early stage of suicide risk. Delineating the mechanisms of risk for PSI in LLD is a crucial direction for late life suicide research. The purpose of our study was to examine whether cognitive flexibility and inhibitory ability are neurocognitive markers of PSI. The secondary purpose of our study was to determine if neurocognitive differences due to PSI are mediated by volumetric differences in the prefrontal cortex.

Methods: Forty community-dwelling middle- and older-aged adults with LLD (18 with PSI, 22 without) completed a neurocognitive battery that assessed cognitive flexibility, inhibitory ability, as well as other neurocognitive domains, and underwent structural neuroimaging.

Results: The PSI group performed significantly worse on cognitive flexibility and inhibitory ability, but not on other neurocognitive tasks which included other measures of executive function. The PSI group had a larger left mid-frontal gyrus (LMFG) than those without PSI, but there was no association between LMFG and cognitive flexibility or inhibitory ability, nor was there statistical evidence of mediation.

Conclusions: Our findings implicate a unique neurocognitive signature in LLD with PSI: poorer cognitive flexibility and poorer inhibitory ability not better accounted for by other domains of cognitive dysfunction and not mediated by volumetric differences in the prefrontal cortex. Volumetric brain differences in the LMFG appear unrelated to differences in cognitive flexibility and inhibitory ability, which suggests two specific but independent risk factors for PSI in middle- and older-aged adults. 